\title{
Fuzzy Logic Approach for Routing in Internet of Things Network
}

\author{
Reena Pingale*, S. N. Shinde
}

\begin{abstract}
A performance of network is evaluated by considering different parameters. The network lifetime depends on many factors Residual energy, Link lifetime and Delay The Major Challenge in loT is to the increased lifetime of low power and lossy network (RPL).The process considering input and output to evaluate Network performance by considering the above factors. The proposed system makes use of FIS (Fuzzy Inference System) for selecting the best path to maximize network lifetime. The outcome obtained by using MATLAB and Network performance is increased. The excellent route is selected if Residual Energy is 194, Link quality is 51.2 and Delay is 1.05 then excellent route quality is $73.4 \%$.
\end{abstract}

Keywords: fuzzy logic; Internet of Things (loT); link lifetime; reliability; residual energy

\section{INTRODUCTION}

The word [1] "Internet of Things (IoT)" performances as an umbrella word that shields the several structures. The deployment of different embedded devices having to sense capabilities to communicate with embedded devices and linking between physical and digital devices . The IoT is providing smarter services and always changing technology [2]. In [3] Internet of Things (IoT) has delivered a encouraging opportunity to form prevailing developed structures and applications by leveraging the increasing ubiquity of radiofrequency identification (RFID), and wireless, mobile, and sensor devices. As an energy to comprehend the enlargement of IoT in productions novelists evaluation the recent exploration of IoT, key-enabling skills, foremost IoT solicitations in productions, and recognizes exploration leanings and encounters. The key involvement of authors is that they brief the recent advanced IoT and its use in businesses analytically.

RPL is measured [4] ordinary for improving the routing structure for congregating troupe movement design. Preliminary from a boundary router, RPL paradigms a DODAG by one or numerous metrics. The DODAG is produced by seeing the concerning budgets, node restriction and multiobjective purpose. Rank group for each node on the DODAG is completed by the detached function. It cares numerous kinds of circulation such as MTP, points to multipoint and points to point. For consuming free topology, the rank essential severely growth from the root near plants of the DODAG. In composite situations lossy relation system is separated into several screens reliant on the request's situation. So in conditions, it might procedure numerous awkward DODAG's with autonomous roots. RPL having many occurrences and it can be route synchronously on the system device and in RPL if nodes need to contribute in DODAG by using different direction-finding procedures for conclusion the greatest way to transporting data. In this paper, we suggest three main limitations residual energy (RE), Link Lifetime (LT), Delay to choice the greatest direction.

The main objective of this research is to invent and enlarge routing algorithm for IoT Network by proposing
Node selection algorithm. The goal of the research is to develop a novel routing strategy based node selection algorithm. The selection of best route is based on residual energy, link quality and delay.

The major contributions of the paper to proposed Node Selection algorithm to acquire best route quality to improve network performance for IoT network.

The section of the paper is planned as follows: Literature survey is considered in section 3 , Section 4 cover the problem definition, section 5 deals with the proposed factors affecting to route. Section 7 with proposed algorithm. The results, with outcome in section 8 and, section 9 deliberates the conclusion.

\section{RELATED WORKS \\ 2.1 RPL Overview}

RPL routing protocol remains to exploit the complete generation of the system by attractive maintenance of the most energy-constrained nodes. RPL planned the Expected ELT for meaning the outstanding instance of the node. They created a DODAG constructed on the ELT metric for precisely approximating the period of all the routes near the boundary router and envisioned a device for observing bottlenecks designed for dispersion the circulation load to numerous parents.

RPL [4] has mostly four control messages, DODAG Information Solicitation (DIS), Information Object (DIO), Advertisement Object (DAO) and Advertisement Object Acknowledgement (DAO-ACK). Firstly, the DODAG request is carried out in two ways

- Applicant node directs the DIS demand to DODAG

- DODAG directs the DIO demand messages to all contributor nodes.

The DODAG permits the drop timer and the contributor node wants to transmit DAO controller communication to DODAG inside the time intermission. Then, the DODAG direct DAO-ACK controller communication to entirely contributor nodes. 


\subsection{Challenges}

1.1 The steady system is conserved by decreased the overhead and end-end delay [5].

1.2 The routing in the system in serious condition due to convergence problems [6].

1.3 The main factors related to security tasks are network topology [7].

1.4 The IoT used mainly the relay function for proper functioning of sensor node.

\section{LITERATURE SURVEY}

Many types of researches have areas completed work on energy-aware routing in RPL and in this, it will minimize energy consumption and increase network lifetime.

In [8] this offerings the routing protocols for the Internet of Things which is supportive in transporting the data into the vapors or to the operators. Several of the general directionfinding protocols are studied in this laterally with the submissions of IoT. In this paper stretches a short-term opinion of the tasks which originate when by IoT for realtime. Here IPv6, CoAP, MQTT and RPL routing protocols are conferred and enlarged. IoT consumes the possible to yield a huge quantity of facts into the folders and the data will be transmitted proficiently.

Secure Multi-hop Routing Protocol (SMRP)[9] protocol attentions on collective the security of the data by avoiding spiteful outbreaks. This direction-finding protocol allows the IoT strategies to confirm previously starting a novel network or construction a standing one. The confirmation uses multilayer restrictions such as User-Controllable ID, user's pre-agreed submission(s) and list of allowable strategies into routing algorithms for joining the confirmation and routing procedures without suffering substantial expenses.

As per observation by Sharief M. A et al. [10], given that IoT system fits to dissimilar holders, PAIR protocol announce a estimating perfect for assistances the transitional nodes to acquire the economic assistance as they apply their properties for transmitting. As estimating perfect of PAIR protocol is based on many restrictions like Residual energy and power consumption, recent weight and buffer space, Distance to neighbours.

The persistence of the routing network designed for IoT (AOMDV-IoT) [11] is to find and generate the linking among expected nodes and the Internet nodes. The protocol defines as reactive protocol that defines the pathway on request. In this paper, the author contributions an expansion of AOMDV improved used for IoT, which can choice a steady Internet broadcast pathway energetically through informing the Internet linking the table. Using reproductions authors presented that the package defeat is better-quality then the end to end delay is reduced.

The main detached of the Energy-aware Ant Routing Algorithm (EARA) is to adjust the routing process for exploiting the lifetime of network [12]. It defines as the swarm intelligence algorithm and reflects the similarly equal number of nodes. As the remaining drive in the IoT strategies deviations finished phase, the authors had announced the instrument near appraise energy evidence. Routing protocol originated on link and residual energy (REL) [13] usages the linkage excellence of remaining energy and wireless network throughout the pathway collection procedure to growth organizations dependability then offers QoS towards the various IoT requests. The load balancing device of this protocol circumvents the extreme use of a solitary track or solitary knot which can additional support in dropping the spots or energy hovels in the system. The energy application will be unchanging in the system. In this paper [14], the authors spoke the network lifespan optimization for the wireless sensor system. The Authors defined the strategy and investigation of numerous energy complementary methods. For a consistent grid topology, we resulting an ideal explanation. The authors demanded that the location of the base position (in the corner) streamlines the optimization problem. They presented that variable the base station location presents new dissimilarities restrictions to the problematic.

Authors in [15] reflect together energy and delay metric to discovery and best pathway with lowest energy ingesting and a lowest end to end delay for real-time circulation in wireless sensor systems. This total is calculated as a linear grouping of the broadcast delay and node's energy on the pathway.

\section{PROBLEM DEFINITION}

Internet of things having an increase in the number of devices due to this strategy traffic will increase which is beyond the capacity of the network. The outcome will be to decrease the performance of the network. It is necessary to find proper routing paths that will give good network performance.

\section{THE PROPOSED WORK}

We suggest an enhanced type of RPL network. The fuzzy logic approach to excellent the finest direction to transmission the facts proficiently. The proposed algorithm finds out the quality of the selected node and it compares with the set of nodes and then selects the finest node in DODAG and the remaining nodes send data through the finest node

The factors consider as below.

\subsection{Residual Energy Consumption}

Residual Energy ingestion of node is calculated after every time interval $t$. With the following equation, it is possible to find out the value of every node with some time interval [16].

$$
\begin{aligned}
& E N_{t}=\left(N_{t} \cdot E_{t}\right)+\left(N_{r} \cdot E_{r}\right), \\
& R_{e_{t}}=\frac{E_{\text {initial }}-E_{\text {spent energy }}}{E_{\text {initial }}},
\end{aligned}
$$

Where: $E N_{t}$ - After time $t$ energy spent by node $N, N_{t}$ - Total of transferred packets, $N_{r}$ - Total of expected packets, $E_{t}$ - 
Energy of transferred packet, $E_{r}$ - Energy of acceptance the packet.

The remaining energy is intended by the variance among primary energy and consumed energy

\subsection{End to End Delay}

As per specified [17] as average interval occupied by data packets to effectively communicating messages crossways the system from source to destination

Delay $=\sum_{i=1}^{n} \frac{\left(R_{i}-S_{i}\right)}{n}$.

\subsection{Link Lifetime}

The system link lifespan is predicated from the quantity of transmissions. It represents forward and reverses data delivery.

$N_{i}(X)=\frac{1}{F_{d} \cdot R_{d}}$.

The Link quality of the path can be calculated by

$N_{q}=\sum_{i=1}^{n} N_{i}(X)$

Where: $N_{i}$ - Link Lifetime, $F_{d}$ - Represents data packet reach to the destination successfully, $R_{d}$ - Represents acknowledge packets are received by the sender successfully [18].

\section{FUZZY LOGIC BASED ROUTING ALGORITHM IN RPL}

The fuzzy logic applies completed routing to excellent the greatest route for transporting data effectively with attention of three-parameter Residual energy consumption, Delay and Link lifetime. The fuzzy logic set was presented in 1965 as a scientific way to denote linguistic vagueness (Zadeh, 1965) [21]. Allowing to the fuzzy logic impression, features and measures can be secret without certain bounds. Fuzzy logic is actual valuable for lecturing real-world difficulties, which typically contain a grade of vagueness.

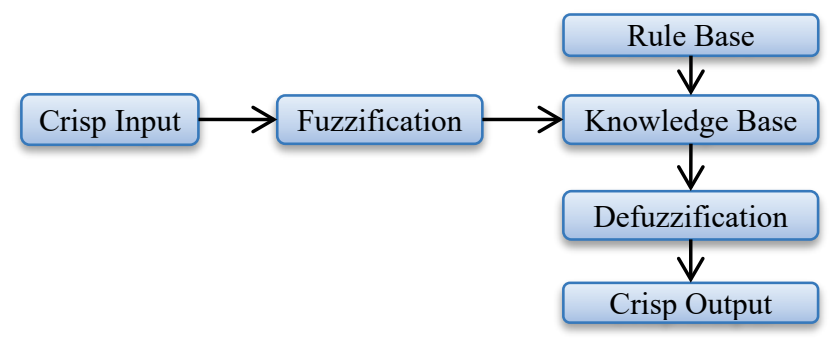

Figure 1 Fuzzy inferences System
The FIS takes linguistic inputs (as stated for simplification), procedures the evidence and outputs the presentation [19].

\subsection{Fuzzification}

Fuzzification takes input fuzzy value from crisp value. The input values are (Residual Energy, Link Lifetime, Delay) its convert these values in linguistic variable and membership function

Table 1 Fuzzy Input Variables

\begin{tabular}{|l|c|}
\hline \multicolumn{1}{|c|}{ Linguistic Expression } & Input Range \\
\hline Low Residual Energy & $0-100$ \\
\hline Average Residual Energy & $50-200$ \\
\hline High Residual Energy & $150-250$ \\
\hline Low Link Lifetime & $0-25$ \\
\hline Average Link Lifetime & $15-80$ \\
\hline High Link Lifetime & $60-100$ \\
\hline Low Delay & $0-2$ \\
\hline Average Delay & $1-4$ \\
\hline High Delay & $3-5$ \\
\hline
\end{tabular}

Table 2 Fuzzy output Variables

\begin{tabular}{|l|c|}
\hline \multicolumn{1}{|c|}{ Linguistic Expression } & Output Range \\
\hline Awful Route Quality & $0-25$ \\
\hline Bad Route Quality & $10-35$ \\
\hline Degraded Route Quality & $25-50$ \\
\hline Average Route Quality & $40-60$ \\
\hline Acceptable Route Quality & $50-75$ \\
\hline Good Route Quality & $60-90$ \\
\hline Excellent Route Quality & $75-100$ \\
\hline
\end{tabular}

\subsection{Linguistic Variable}

The variable represents the input and output of the variable. In this residual energy having three linguistic variables High, Average and Low. The output variable also define linguistic variable Awful, Bad, Degraded, Average, Acceptable, Good, Excellent

\subsection{Membership Function}

It is a mapping of membership function values to the real world measurement values, so that the actions can be functional to them. This function evaluates the linguistic variable. Membership function values are in-between range 0 to 1.

$\mu_{A}(z)=\left\{\begin{array}{ccc}1 & \text { if } & z \leq a \\ \frac{z-a}{x-a} & \text { if } & a<z \leq x . \\ 0 & \text { if } & z \geq x\end{array}\right.$

\subsection{Fuzzy Rule Base}

The effect which the FIS types is resulting from the instructions which are kept in the record. These are kept as a set of instructions. The rules are 'If-Then' declarations that are in-built and informal to appreciate meanwhile they are unknown but public English declarations 


\subsection{Defuzzification}

It is the procedure of changing the fuzzy input into a crisp set. The value ranges by MF in between 0 and 100 and it delivers single crisp value. We require certain weighted average technique for Defuzzification [20].

$$
X=\frac{\sum_{i=1}^{n} Z_{i} \cdot \mu \cdot B\left(Z_{i}\right)}{\sum_{i=1}^{n} \mu \cdot B\left(Z_{i}\right)} .
$$

The fuzzy inference system to determine the optimal path from a basis node to the endpoint node. This will progress the performance of the network.

\section{PROPOSED WORK BASED ON RANK CALCULATION}

The rank of the node computes from the root node and at each level increases the rank by 1 . The Equation value can be calculated by using the Defuzzification process. The rank equation can be defined as

Rank1 $(N)=$ Root_Rank $(N)$ + Increase_Rak1 Increase_Rak1 $=\overline{\text { Equation }}+$ MinHop_Rank_increase

\subsection{Node Selection Process}

The node selection process based on construction of MF using rule based system. The node selection process using FIS system.

Algorithm1 - Node Selection

\begin{tabular}{|c|c|}
\hline & $\begin{array}{l}\text { INPUT: No.of Node N, Node_parennt_ID, Sender_parentID, } \\
\text { Excellent Route }=\infty\end{array}$ \\
\hline 2. & Output: Selected Route \\
\hline & For Selected_Node $\in$ List_Node do \\
\hline & Rank (No. of. Node $N$ ) $<-$ Rank_Selected_Node $(N)+$ Increase_Rank \\
\hline & Increase_Rak1 $<-$ Equation + MinHop_Rank_increase \\
\hline & Intialize variable Energy, Link_quality, Delay \\
\hline & Consider fuzzy input and output \\
\hline & $\begin{array}{l}\text { Input : No. of Node } N \text {, Node_parennt_ID, Sender_parent_ID, } \\
\text { Excellent Route }=\infty\end{array}$ \\
\hline & Output : Selected Route \\
\hline & Contruct fuzzy membership_function \\
\hline & Check fuzzy rule base in fuzzy inference system \\
\hline & Apply Defuzzification \\
\hline & Equation $=\frac{\sum_{i=1}^{n} Z_{i} \cdot \mu \cdot B\left(Z_{i}\right)}{\sum_{i=1}^{n} \mu \cdot B\left(Z_{i}\right)}$ \\
\hline & If Excellent Route $>=$ Selected Route then \\
\hline & Excellent Route $<-$ Selected Route \\
\hline & end if \\
\hline & \\
\hline
\end{tabular}

\subsection{Results and Discussion}

This segment demonstrates the evaluation of anticipated system with fuzzy inference over feigning the routing for IoT network. The analysis is done by Fuzzy rule based system.

The study of presentation built on the suggested Node Selection algorithm using residual energy, delay and link quality factors is estimated in this segment with output parameter as Route quality.

The analysis is performed by selecting rule based system using FIS to generate the result. The analysis is done by varying parameters

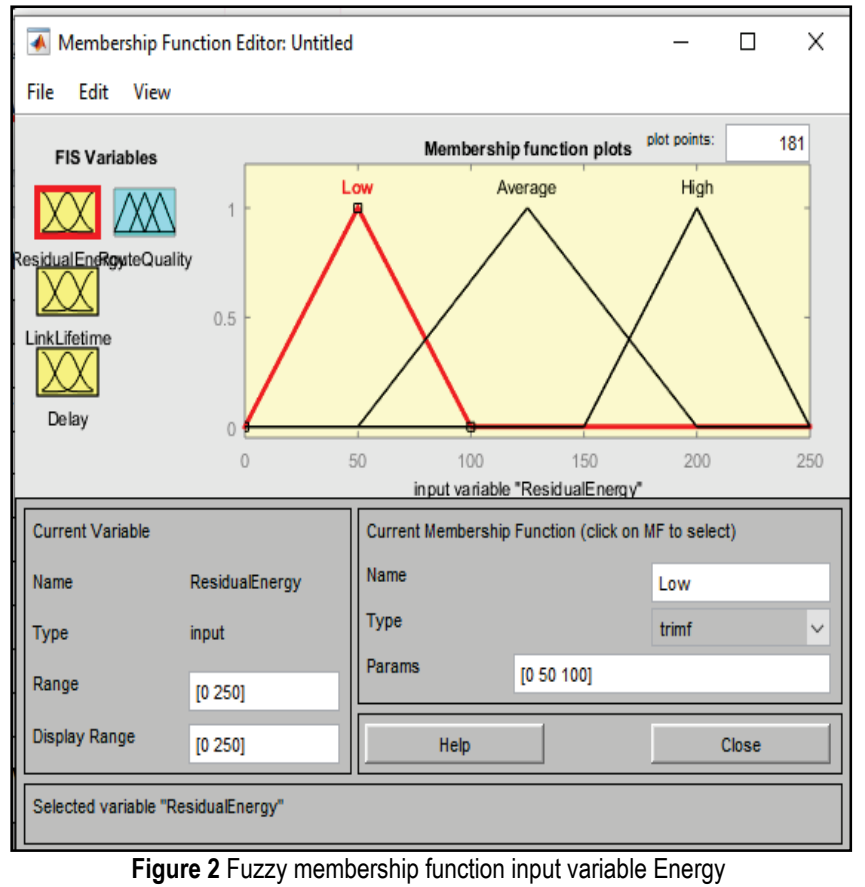

The Residual energy values are represented in between 0 to 250. The linguistic parameters are Low, Average and High. The membership function collections from 0 to 1.If the Network lifetime increases then the network is energy efficient. The fuzzy membership function can be represented of input variable delay, residual energy and link quality and output variable representation using a fuzzy rule-based system. The delay values are represented in between 0 to 5 and Link quality values are 0 to 100 .

The fuzzy set is grouping of dissimilar metrics, every metric cover specific fuzzy variable. The rule constructed contains of $3^{3}=27$ fuzzy based rules. This is constructed on the input variable and membership function. We can describe the fuzzy based rule which characterizes the first column as the count of total number of rules and next 2 to 4 column signifies input fuzzy logic variable and the last column characterizes output variable in the form of Route Quality. The output follows max operator as combination and min operator as configuration function. 


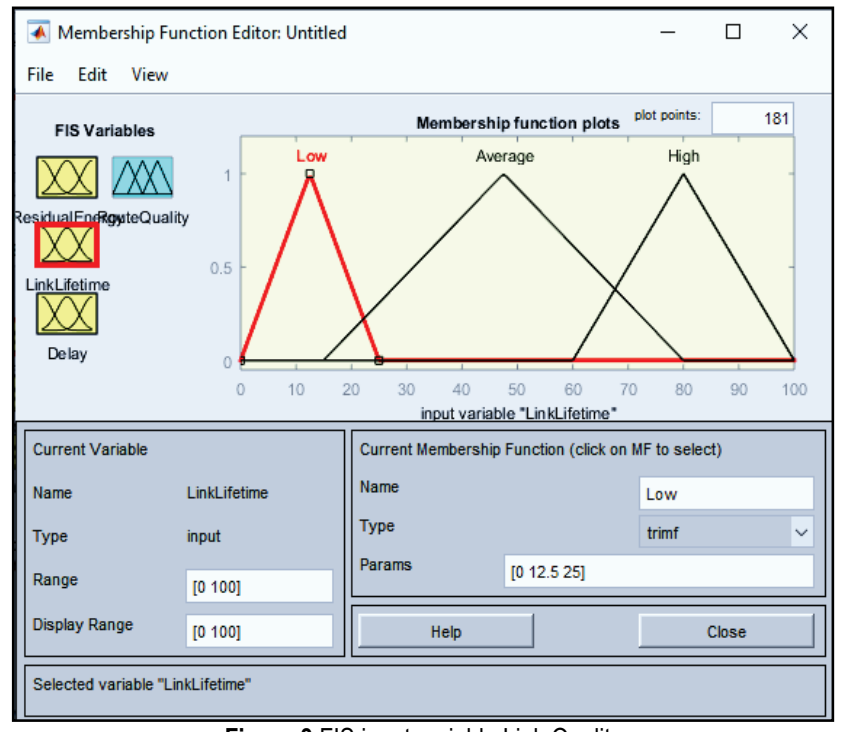

Figure 3 FIS input variable Link Quality

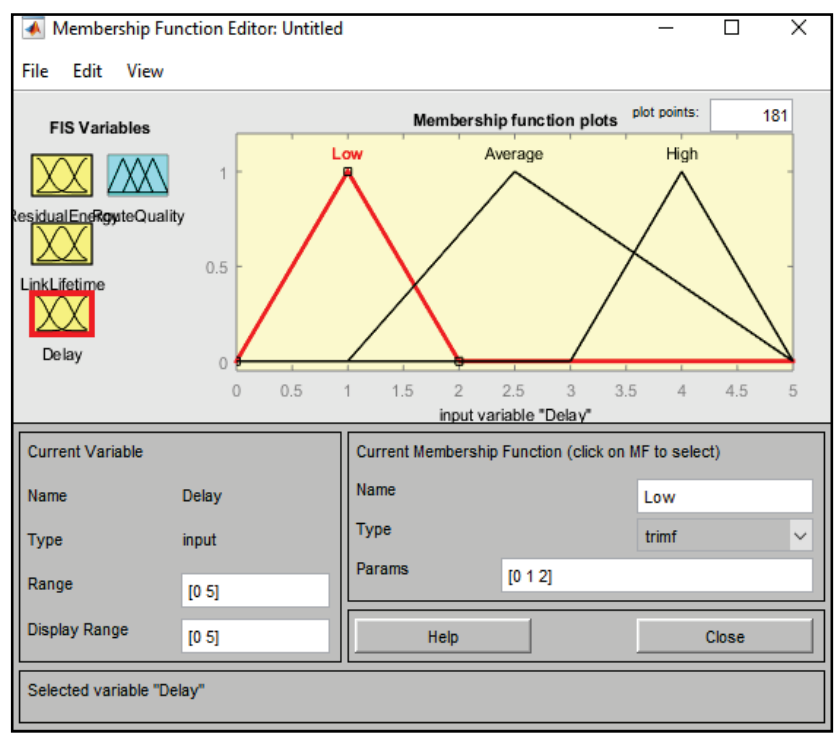

Figure 4 FIS input variable Delay

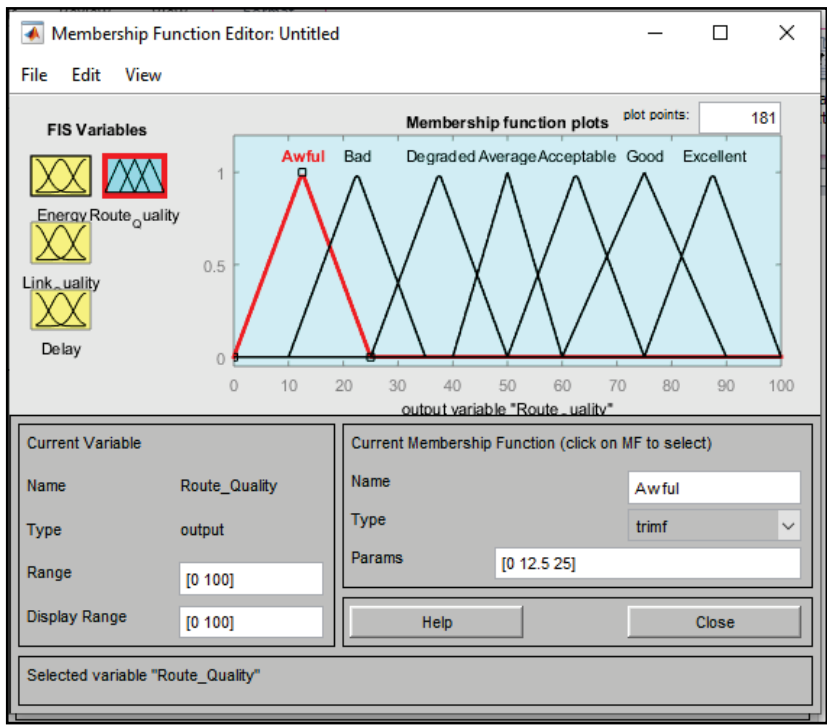

Figure 5 FIS output variables Route Quality

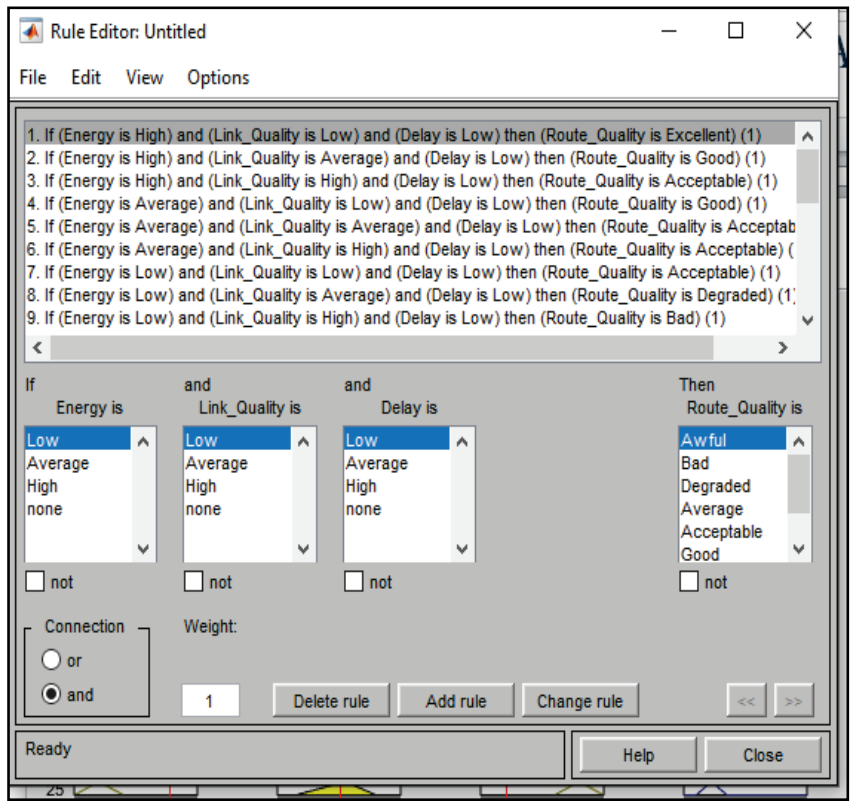

Figure 6 Fuzzy Rule-based system

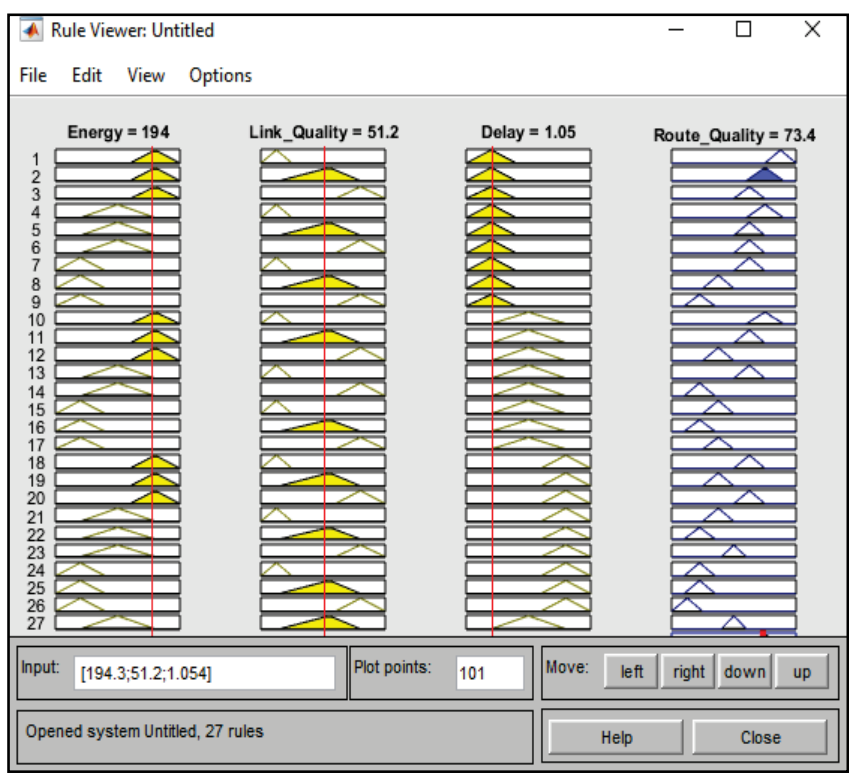

Figure 7 Outcome of Route Quality

From Fig. 7, we observed that Route quality is above 73.4\% means its excellent route selected from this we first declare the variable as Residual Energy is 194, Link Quality is 51.2 , and Delay is 1.05 . As per fuzzy membership function, the linguistic variable Energy value is "Average and high" and MF values are 0.5 and 0.5 . The Link Quality of the linguistic variable significance is "Average" with MF value is 1 , linguistic variable significance of delay is "Low" with the MF value is 1 . From rule number (2) and (5) its process outcome in the form of route quality parameter as acceptable and good. The Defuzzification process can be applied and calculated using a formula

$$
X=\frac{(0.5 \cdot 68+0.5 \cdot 79)}{0.5+0.5}=73.4
$$


From the outcome excellent route selected with proper selection of input variable. The surface view can be represented with parameter detail.

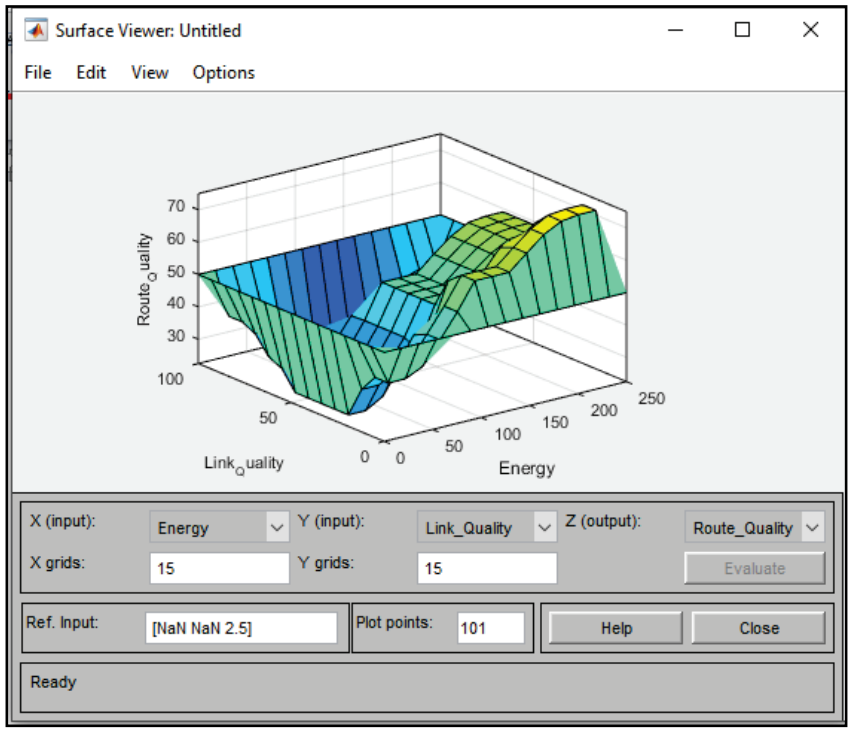

Figure 8 Surface Residual Energy, Link Quality, Delay

\section{CONCLUSION}

In this paper fuzzy logic approach for RPL network utilised for the IoT network system. Considering the input and output parameter in a FIS to generate the required outcome in the form of route quality. The selection of the route is constructed on three factors Residual Energy, Link Lifetime, Delay to generate proper route selection to increase network lifetime. The suggested algorithm allows the operative presentation and collection of achievable and excellent path. The yield of anticipated algorithm is calculated by selecting excellent route, if Residual Energy is 194, Link quality is 51.2 and Delay is 1.05 then excellent route quality is $73.4 \%$. The Matlab simulation gives the outcome in the form of route quality and future work will consider the deployment of the node in the real-time environment.

\section{Notice}

This paper was presented at IC2ST-2021 - International Conference on Convergence of Smart Technologies. This conference was organized in Pune, India by Aspire Research Foundation, January 9-10, 2021. The paper will not be published anywhere else.

\section{REFERENCES}

[1] Miorandi, D., Sicari, S., De Pellegrini, F., \& Chlamtac, I. (2012). Internet of things: Vision, applications and research challenges. Ad Hoc Networks, 10(7), 1497-1516. https://doi.org/10.1016/j.adhoc.2012.02.016

[2] Coetzee, L. \& Eksteen, J. (2011). The Internet of Things -Promise for the future? An introduction. In IST-Africa Conference Proceedings, 2011, 1-9.
[3] Li Da Xu, Wu He, \& Shancang Li. (2014). Internet of Things in Industries: A Survey. IEEE Transactions on Industrial, Informatics, 10(4), 2233-2243. https://doi.org/10.1109/TII.2014.2300753

[4] Thubert, P., Winter, T., Brandt, A., Hui, J., Kelsey, R., Levis, P., Pister, K., Struik, R., Vasseur, J., \& Alexander, R. (2012). RPL: IPv6 Routing Protocol for Low power and Lossy Networks. IETF. RFC 6550.

[5] Lim, W. H. \& Isa, N. A. M. (2014). Particle swarm optimization with adaptive time-varying topology connectivity. Applied Soft Computing, 24, 623-642. https://doi.org/10.1016/j.asoc.2014.08.013

[6] Quan Le, Thu Ngo-Quynh, \& Magedanz, T. (2014). RPL-based multipath routing protocols for internet of things on wireless sensor networks. In 2014 International Conference on Advanced Technologies for Communications (ATC 2014), 424429.

[7] Al-Turjman, Fadi. (2017). Energy-aware data delivery framework for safety-oriented mobile IoT. IEEE Sensors Journal, 18(1), 470-478. https://doi.org/ 10.1109/JSEN.2017.2761396

[8] Channamma, S., Bhuvaneshwari, M., Pooja, M., \& Jayalakshmi, J. (2016). Routing, Application and Research challenges in IoT. Special Issue on ICRIET-2016, Published by Research Trend, Website: www.researchtrend.net

[9] Loh, P., Chze, R., \& Leong, K. S. (2014). A Secure Multi-Hop Routing for IoT Communication. IEEE World Forum on the Internet of Things (WF-IoT), 428-432. https://doi.org/10.1109/WF-loT.2014.6803204

[10] Oteafy, S. M. A., Al-Turjman, F. M., \& Hassanein, H. S. (2012). Pruned Adaptive Routing in the Heterogeneous Internet of Things. Global Communications Conference (GLOBECOM), 2012 IEEE, 214-219. https://doi.org/10.1109/GLOCOM.2012.6503115

[11] Tian, Y. \& Hou, R. (2010). An Improved AOMDV Routing Protocol for the Internet of Things. 2010 International Conference on Computational Intelligence and Software Engineering (CSE 2010), Wuhan, 1-4. https://doi.org/10.1109/CISE.2010.5676940

[12] Frey, M., Grose, F., \& Gunes, M. (2014). Energy-aware Ant Routing in Wireless Multihop Networks. 2014 IEEE International Conference on Communications (ICC 2014), 190-196. https://doi.org/10.1109/ICC.2014.6883317

[13] Machado, K., Rosário, D., Cerqueira, E., Loureiro, A. A. F., Neto, A., \& de Souza, J. N. (2013). A Routing Protocol Based on Energy and Link Quality for Internet of Things Applications. Sensors, 13(2), 1942-1964. https://doi.org/10.3390/s130201942

[14] Kacimi, R., Dhaou, R., \& Beylot, A.-L. (2013). LoadBalancing Strategies for Lifetime Maximizing in Wireless Sensor Networks. Ad Hoc Networks, 11(8), 2172-2186. https://doi.org/10.1016/j.adhoc.2013.04.009

[15] Mohajerzadeh, A. H. \& Yaghmaee, M. H. (2009). An efficient energy-aware routing protocol for real-time traffic in wireless sensor networks. International Conference on Ultra Modern Telecommunications \& Workshops, ICUMT '09, 1-9. https://doi.org/10.1109/ICUMT.2009.5345536

[16] Hu, X., Wang, J., \& Wang, C. (2011). Mobility-adaptive routing for stable transmission in mobile ad hoc networks. Journal of Communications, 6(1), 79-86.

[17] Douglas, S. J., de Couto, D., Aguayo, J., \& Bicket, R. M. (2003). A High-Throughput Path Metric for Multi-Hop Wireless Routing. Wireless Networks, 11(4), 419-434. https://doi.org/10.1145/938985.939000 
[18] The Mathworks. (2009). Fuzzy Logic Toolbox User's Guide, The Mathworks Inc. Retrieved: September 10, 2009 from http://www.mathworks.com/access/helpdesk/help/pdf_doc/fuz zy/fuzzy.pdf.

[19] Alhumaidi Hanouf, M. (2016). Fuzzy weighted average approach to ranking projects in contractor initial bidding. Fuzzy information processing Society, 2016 Annual conference of the North American, IEEE, 1-7.

[20] El Alami, H. \& Najid, A. (2017). Fuzzy Logic Based Clustering Algorithm for Wireless Sensor Networks. International Journal of Fuzzy System Applications (IJFSA), 6(4), 63-82. https://doi.org/10.4018/IJFSA.2017100105

[21] Zadeh, L. A. (1965). Fuzzy sets. Information and Control, 8(3), 338-353. https://doi.org/10.1016/S0019-9958(65)90241-X

\section{Authors' contacts:}

Ms. Reena P. Pingale, Assistant Professor

(Corresponding author)

Sinhgad College of Science,

S. No. 9/1/5 and 9/2/4, Off West. Bypass Highway,

Ambegaon (BK.), Pune - 411041, Maharashtra, India

reena.pingale@gmail.com

Dr. S. N. Shinde, Principal

C.M.C.S. College

Udoji Maratha Boarding Campus, Near, Gangapur Road,

D. K. Nagar, Nashik, Maharashtra 422013, India

sns110@gmail.com 